\title{
Knowledge and Attitude Regarding Child Abuse Among Primary Healthcare Physicians and Interns in Al Qassim, Saudi Arabia
}

Abdulrhman Aldukhayel ${ }^{1}$, Emad Aljarbou ${ }^{2}$, Fatima M. Alturki ${ }^{2}$, Nouf S. Almazyad ${ }^{2}$, Ohud M. Alsaqer ${ }^{2}$, Raghad Almutairi ${ }^{2}$

1. Department of Family and Community Medicine, Qassim University, College of Medicine, Buraidah, SAU 2. Medicine, Qassim University, College of Medicine, Buraidah, SAU

Corresponding author: Abdulrhman Aldukhayel, aaldukhayel@qumed.edu.sa

\section{Abstract}

\section{Introduction}

Child abuse can include physical abuse, psychological abuse, sexual assault, neglect, or failure to meet the child's basic needs. It can lead to major psychosocial problems resulting in long-lasting consequences for the child.

\section{Objective}

This study aimed to assess the knowledge and attitude (KA) of primary healthcare physicians and interns regarding child abuse in Al Qassim, Saudi Arabia.

\section{Materials and methods}

This multicenter, cross-sectional study was conducted among primary healthcare physicians and interns in the Qassim region, Saudi Arabia, from July to October 2020. A self-administered questionnaire was distributed among the targeted physicians using either an online platform or face-to-face interviews. The questionnaire was devised from the pre-tested literature and formal discussions with experts. A total of 292 primary healthcare physicians and interns participated in our study. A consent form and brief details of the study were given ahead of the recruitment. Data were tabulated in a Microsoft Excel spreadsheet (Microsoft Corporation, Redmond, WA); a separate codebook was prepared with a description of the variables and corresponding codes, and all statistical analyses were performed using IBM SPSS Statistics for Windows, Version 21.0 (IBM Corp., Armonk, NY).

\section{Results}

A total of 292 respondents were involved in the study. Among them, 45\% ( $n=131)$ were medical interns, $39.4 \%(n=115)$ were residents, and the rest were either specialists ( $n=34 ; 11.6 \%)$ or consultants $(n=12 ; 4.1 \%)$. Among the respondents, the KA level was found to be moderate, high, and low in $68.8 \%, 28.4 \%$, and $2.7 \%$, respectively. The overall mean KA score was 81.1 [standard deviation (SD): 9.56] out of a possible 115 points. Factors associated with high KA were age ( $>30$ years), non-Saudi nationality, having children, and having studied outside Saudi Arabia; The only factor significantly associated with low levels of KA was being a pediatrician $(\mathrm{p}<0.001)$.

\section{Conclusion}

Overall, the participants displayed adequate levels of KA regarding child abuse. Better KA was observed among expatriate physicians who were parents themselves and had earned their degrees outside the Kingdom of Saudi Arabia. However, pediatricians demonstrated poor knowledge with regard to child abuse.

Categories: Family/General Practice, Pediatrics, Psychiatry

Keywords: child abuse, neglect, attitude, knowledge

\section{Introduction}

Child abuse encompasses physical abuse, psychological abuse, sexual assault, and/or neglect and failure to meet the child's basic needs. It can lead to major social problems resulting in long-lasting consequences for the child [1]. However, cultural differences around the world concerning child discipline and parenting comprise a wide range of approaches making a clear, singular definition of child abuse or neglect challenging to establish [2]. In 1990, the first case of child abuse was officially recorded in Saudi Arabia, and in 1994, a government committee was formed to oversee the protection of children's rights in the country [3]. Physicians are obligated to report cases of child abuse to the concerned social welfare organizations or government authorities to provide early interventions for abuse victims to prevent further abuse [4]. 
Physicians can use screening instruments for assistance in recognizing child abuse at an early stage, thereby helping to prevent any further harm [5]. Despite the obligation of reporting suspected cases of child abuse to child protective services, a 2015 Swedish study reported that more than $50 \%$ of pediatricians and general practitioners did not report cases of child abuse [6]. Moreover, $60 \%$ of general practitioners were not familiar with child abuse guidelines or local protocols within their clinics for handling such situations [6]. A 2017 study in Turkey reported that only $13 \%$ of physicians in their study received training on reporting child abuse [7]. Most physicians in that study (72\%) knew of existing laws to protect children from abuse; however, the authors found that physicians did not report suspected child abuse cases due to a lack of knowledge about the procedures and a desire to avoid involvement in legal procedures [7]. A 2019 study in Saudi Arabia that included all physicians in the Abha region found that there was sufficient awareness regarding signs of suspected cases of child abuse among physicians, but cases were still underreported due to a lack of knowledge about the reporting process and also due to community barriers [8].

In light of this, we conducted this study to assess the knowledge and attitude (KA) among primary healthcare physicians and interns regarding child abuse in Al Qassim, Saudi Arabia.

\section{Materials And Methods}

We conducted a multicenter, cross-sectional study among primary healthcare physicians and interns in the Qassim region of Saudi Arabia from July to October 2020. A self-administered questionnaire was distributed among the targeted physicians using either an online platform or via face-to-face interviews. The questionnaire was devised from the literature and with input from experts via formal discussions. A total of 292 primary healthcare physicians and interns participated in our study. Participants provided written informed consent before inclusion in the study. Responses were collected and tabulated in a Microsoft Excel spreadsheet (Microsoft Corporation, Redmond, WA).

\section{Scoring}

The criteria for KA regarding child abuse were encapsulated in 23 questions (Table 1) with the provision to provide answers through a 5-point Likert scale, with answer options ranging from "strongly disagree" coded as 1 to "strongly agree" coded as 5 . The questionnaire consisted of four domains: knowledge about child abuse and neglect (CAN; eight questions), reasons why child abuse is underreported in Saudi Arabia (five questions), acts of abuse toward children (five questions), and acts of child neglect (five questions). The total KA score was calculated by summing up the answers to all 23 questions, with a possible score range of 23115 , with higher scores indicating higher levels of KA regarding child abuse. Using $50 \%$ and $75 \%$ of the total score points to determine the level of KA, participants were classified as having low KA if the score was from 23 to 58 points; scores of 59 to 86 were considered as moderate KA, and scores of 87 to 115 were classified as high KA. 


\section{Cureus}

Statement

Score, mean \pm SD (out of 5 )

Knowledge about CAN

1. I have enough training to deal with CAN

2. We need to redefine CAN in Saudi Arabia according to our culture and religion

3. The present supportive services to deal with CAN in Saudi Arabia are adequate

4. I prefer resolving a case of child abuse myself rather than reporting it to the police

5. I am aware of the reporting procedures regarding child abuse in my hospital in Saudi Arabia

6. I am willing to report all suspected child abuse cases

7. I prefer limiting my reporting of child abuse to life-threatening injuries

8. Child abuse is underreported in Saudi Arabia

Reasons why child abuse is underreported in Saudi Arabia

9. It is not legally mandatory to report child abuse

10. Reporting might not be good for the sake of the child

11. Reporting procedures are unclear

12. Reporting child abuse to authorities is not yet acceptable in our community

13. Fear of parent response

Acts of abuse toward children

14. Burning the child for misbehavior

15. Locking the child alone at home

16. Severe beating that leaves marks on the child's body

17. Parents throwing different objects at the child when angry

18. Parents smoking in the presence of the child

Acts of child neglect

19. Parents refusing to send the child to school

20. Parents refusing medical treatment or surgical intervention necessary for their child

21. Child with severe dental problems, which are not treated

22. Parents paying no attention to the child's cleanness

23. Child failing to thrive due to social deprivation
$4.12 \pm 0.97$

$219(75.0 \%)$

$2.85 \pm 1.07$

$78(26.7 \%)$

$3.74 \pm 1.22$

199 (68.2\%)

$2.78 \pm 1.04$

$60(20.5 \%)$

$2.25 \pm 1.18$

$53(18.2 \%)$

$2.85 \pm 1.16$

$88(30.1 \%)$

$3.94 \pm 1.04$

204 (69.9\%)

$2.24 \pm 1.27$

$61(20.9 \%)$

$3.56 \pm 1.11$

$156(53.4 \%)$

$2.71 \pm 1.11$

$81(27.7 \%)$

$3.21 \pm 1.38$

$136(46.6 \%)$

$3.46 \pm 1.11$

$149(51.0 \%)$

$3.50 \pm 1.10$

$162(55.5 \%)$

$3.52 \pm 1.07$

162 (55.5\%)

$4.11 \pm 0.95$

$212(72.6 \%)$

$3.90 \pm 0.97$

$205(70.2 \%)$

$4.16 \pm 0.93$

217 (74.3\%)

$4.00 \pm 0.98$

$211(72.3 \%)$

$4.01 \pm 0.85$

$220(75.3 \%)$

$4.08 \pm 0.94$

217 (74.3\%)

$4.02 \pm 0.88$

$222(76.0 \%)$

$3.98 \pm 0.91$

217 (74.3\%)

$4.07 \pm 0.90$

$217(74.3 \%)$

TABLE 1: Assessment of knowledge and attitude regarding child abuse and neglect $(\mathrm{n}=292)$

CAN: child abuse and neglect; SD: standard deviation

\section{Statistical analysis}

Descriptive statistics were demonstrated using numbers, percentages, mean, and standard deviation (SD), as appropriate. Between comparisons, the Mann-Whitney U test and the Kruskal-Wallis test were applied. A pvalue of $<0.05$ was considered statistically significant. Normality tests were conducted using the ShapiroWilk test. All statistical analyses were carried out using IBM SPSS Statistics for Windows, Version 21.0 (IBM Corp., Armonk, NY).

\section{Results}

This cross-sectional study enrolled 292 primary healthcare physicians and interns across the Qassim region. Table 2 presents the sociodemographic characteristics of the respondents. Most respondents belonged to the 


\section{Cureus}

age group of 22-30 years (64.3\%); more than half (56.5\%) of them were men, and approximately two-thirds (66.1\%) were Saudis. Regarding their professional status, $44.9 \%$ were medical interns, and 39.4\% were residents. Furthermore, $40.1 \%$ were married, with approximately two-thirds (64.7\%) having no children. With regard to specialty, nearly $45 \%$ were internists, while $21.2 \%$ were general practitioners. Additionally, nearly two-thirds (65.1\%) of the respondents had received their education within Saudi Arabia, with $63.7 \%$ having practiced medicine for fewer than five years.

Study variables

Age group

22-30 years

$31-40$ years

41-50 years

$>50$ years

Gender

Male

Female

Nationality

Saudi

Non-Saudi

Professional level

Medical Intern

Resident

Consultant

Specialist

Married

Yes

No

Children

None

$1-2$

$>2$

Specialty

General practitioner

Family medicine

Pediatrics

Internist

Other

Country of medical education

Saudi Arabia

Other Arab countries

European country

Others
$190(65.1 \%)$

$84(28.8 \%)$

$03(01.0 \%)$

N (\%)

$188(64.3 \%)$

42 (14.4\%)

39 (13.4\%)

$23(07.9 \%)$

$165(56.5 \%)$

$127(43.5 \%)$

$193(66.1 \%)$

99 (33.9\%)

$131(44.9 \%)$

$115(39.4 \%)$

$12(04.1 \%)$

$34(11.6 \%)$

$117(40.1 \%)$

175 (59.9\%)

189 (64.7\%)

35 (12.0\%)

$68(23.3 \%)$

$62(21.2 \%)$

48 (16.4\%)

$25(08.6 \%)$

131 (44.9\%)

26 (08.9\%)

15 (05.1\%) 


\section{Cureus}

Years in practice

$<5$ years

$186(63.7 \%)$

5-10 years

$36(12.3 \%)$

$>10$ years

$70(24.0 \%)$

TABLE 2: Sociodemographic characteristics of physicians $(n=292)$

KA was divided into four domains: knowledge about CAN, reasons why child abuse is underreported in Saudi Arabia, acts of abuse toward children, and acts of child neglect. The score ascribed to answer options ranged from 1 to 5 points, where a score of five indicated the highest level of awareness. Firstly, regarding knowledge about CAN, respondents showed good knowledge with respect to the following statements: "I am willing to report all suspected child abuse cases" (mean: 3.94), "We need to redefine CAN in Saudi Arabia according to our culture and religion" (mean: 3.74), and "Child abuse is underreported in Saudi Arabia" (mean: 3.56). Respondents' knowledge was low with regard to the following statements: "I prefer limiting my reporting of child abuse to life-threatening injuries" (mean: 2.24) and "I prefer resolving a case of child abuse myself rather than reporting it to police" (mean: 2.25). Secondly, regarding the category of reasons why child abuse is underreported in Saudi Arabia, respondents showed good KA with respect to the following sentences: "Fear of parent response" (mean: 3.52) and "Reporting child abuse to authorities is not yet acceptable in our community" (mean: 3.50). KA was low regarding the statement "It is not legally mandatory to report the child abuse" (mean: 2.71). Thirdly, for the acts of abuse toward children, respondents showed high KA regarding most of the statements, especially with respect to "Severe beating that leaves marks on the child body" (mean: 4.16) and "Burning the child for misbehavior" (mean: 4.11). Finally, related to the acts of child neglect, respondents also showed high KA with respect to most of the statements, and it was really noteworthy pertaining to the statements "Parents refusing to send the child to school" (mean: 4.12) followed by "Parents refusing medical treatment or surgical intervention necessary for their child" (mean: 4.08) and "Child failing to thrive due to social deprivation" (mean: 4.07).

Table 3 summarizes the descriptive statistics of the KA regarding CAN, including the mean score of knowledge about CAN (24.2), reasons why child abuse is underreported (16.4), acts of abuse toward children (20.2), and acts of child neglect (20.3). The overall mean score was 81.1 (SD: 9.56).

Based on the given criteria, we found a low level of CAN KA in $2.7 \%$ of respondents, a moderate level of KA in $68.8 \%$, and a high level of KA in $28.4 \%$ of respondents.

\begin{tabular}{|c|c|c|}
\hline Variables & Number of items & Mean \pm SD \\
\hline Knowledge about CAN & 08 & $24.2 \pm 4.27$ \\
\hline Reasons why child abuse is underreported in Saudi Arabia & 05 & $16.4 \pm 3.98$ \\
\hline Acts of abuse toward children & 05 & $20.2 \pm 3.81$ \\
\hline Acts of child neglect & 05 & $20.3 \pm 3.97$ \\
\hline Total score & 23 & $81.1 \pm 9.56$ \\
\hline
\end{tabular}

TABLE 3: Descriptive statistics of the knowledge and attitude regarding CAN ( $\mathrm{n}=292)$

CAN: child abuse and neglect; SD: standard deviation

When measuring the differences between the KA scores in relation to physician sociodemographics, the KA of those in the older age group ( $>30$ years; $\mathrm{T}=-2.723 ; \mathrm{p}=0.025)$, non-Saudis $(\mathrm{T}=-3.451 ; \mathrm{p}=0.002)$, those who have children $(\mathrm{T}=2.465 ; \mathrm{p}=0.031)$, and those who had earned their degree outside of Saudi Arabia $(\mathrm{T}=-2.911$; $\mathrm{p}=0.005$ ) was significantly higher than their counterparts. Those in pediatric specialty showed significantly lower KA scores compared to those in other specialties (F8.274; $<<0.001$; Table 4). On the other hand, gender, professional level, marital status, and years in practice did not significantly affect the KA score (all $\mathrm{p}>0.05)$.

\section{Discussion}

CAN is documented in every country and culture, and child maltreatment is associated with several 
consequences, including parental depression, stress, and social isolation [9]. The protection of children is essential, and suspected child abuse cases should be reported and documented to the concerned authorities who can assess the situation and help the young ones [9]. Appropriate investigation and intervention to help the abuse victims are necessary to alleviate the trauma of these children and their families. The purpose of the present study was to determine the KA among primary healthcare physicians regarding child abuse.

Unfortunately, there appear to be gaps in the system that allows for underreporting of abuse cases, and the knowledge levels are not consistent: several studies have reported adequate knowledge regarding child abuse among physicians [8,10-13], while other studies report insufficient knowledge levels [14,15]. A long-term study investigating potential child abuse among all children younger than 18 years of age showed that the annual prevalence of child abuse is $1 \%$, with emotional neglect being the most common type [16]. A study in France in 2015 on the level of knowledge about diagnosing child abuse among family physicians showed that $30 \%$ had previous training for dealing with abuse cases [17]. In that study, $82 \%$ of physicians reported having suspected cases of child abuse. The study showed that the main obstacles to reporting such cases were the fear of misdiagnosis and the fear of separating the children from their families with a lack of knowledge about the reporting process [8]. Sathiadas et al. [18] reported that although healthcare professionals demonstrated good overall knowledge, $65.8 \%$ of physicians were unsatisfied with their knowledge and expressed the desire for some form of education on child maltreatment to further their understanding. AlBakr et al. [16] reported that the overall knowledge among all primary healthcare physicians in Al-Khobar City in the Eastern Region of Saudi Arabia about child abuse was significantly higher among those aged 3640 years, which aligned with our findings related to the age of the respondents. They also reported that longer experience was associated with higher levels of knowledge [16]; however, we did not find this to be true in our study as we found no significant association between the years in practice and the overall KA score.

We found that those who had earned their degree outside Saudi Arabia had better KA scores than those who studied within the Kingdom, This contrasts with the findings of Habib [13], who found that respondents receiving their medical education in Saudi Arabia scored statistically significantly higher in CAN knowledge regarding "professional experience about child abuse and negligence" compared to pediatricians who completed their medical education in Western countries. Furthermore, Habib [13] indicated that gender differences were not significant, which was consistent with our results.

In Iran, investigators have reported that pedodontists had higher levels of knowledge about CAN than general dentists [12]. This did not seem to disagree with our results, as we observed that specialty significantly influences the knowledge score, with pediatricians exhibiting lower knowledge scores than doctors in other specialties. However, our results contradict the report by Sathiadas et al., who found that the knowledge, attitude, and behavior of healthcare professionals toward child abuse were significantly good among respondents within the pediatric specialty [18]. Similarly, we also noted that physicians with children have significantly better KA scores than those without children.

Moreover, it is essential to mention that more than $53 \%$ of the physicians agreed that child abuse was underreported within Saudi Arabia. This is consistent with the findings of Alsaleem et al., who reported that $64 \%$ of primary healthcare physicians noticed underreported child abuse [10]. Alnasser et al. emphasized that child maltreatment in Saudi Arabia was common [12]. Several factors have been attributed to the underreporting of child abuse. In our study, the most commonly mentioned reason for underreported instances of child maltreatment was fear of parent response followed by the feeling that reporting of child abuse is not yet acceptable and reporting procedures are not clear, which was also in accordance with the findings of Alsaleem et al. [10]. Also, approximately $70 \%$ of physicians thought it is important to redefine CAN in Saudi Arabia according to the country's culture and religion, which was strikingly similar to the findings of Alsaleem et al. [10].

There are several limitations to this study. Specifically, the study was conducted in a single region in Saudi Arabia. Therefore, the results may not be generalizable, replicable, or even applicable to other regions. This implies that the convenience sampling method has the potential for sampling bias. Additionally, this study design may have introduced some recall bias and subjectivity.

\section{Conclusions}

Most of the participants in this study showed adequate KA regarding CAN. High KA scores were seen among expatriate physicians who were parents and older than 30 years in age. Pediatricians demonstrated poor KA with regard to CAN. It is necessary to address the gaps in KA regarding CAN, most especially among pediatricians. Given that pediatricians are primarily in charge of children's health and wellness, they need to have adequate knowledge and information about CAN. More CAN courses and training should be implemented to address the lack of KA regarding CAN among pediatricians.

\section{Appendices}

Knowledge and Attitude regarding child abuse among primary healthcare physician in Qassim, Saudi Arabia 


\section{Cureus}

This questionnaire aims to assess your knowledge and attitude regarding child abuse. its results will help us to improve this aspect.

1. Gender

male

female

2. Age:

3. Married

Yes

No

4. No. of children:

5. Nationality

Saudi

Non-Saudi

6. Education level

Resident

Specialist

Consultant

Other

7. Specialty

GP

Family medicine

Pediatrics

Internist

Other...

8. Country of medical education

Saudi Arabia 


\section{Cureus}

Arab country

European country

North America

Other

9.Years of practice:

Kindly score the fowling items regarding child abuse and neglect out of 5 as follows: Strongly disagree: 1, Disagree: 2, Neutral: 3 , Agree: 4, Strongly agree: 5

I have enough training to deal with child abuse and neglect

We need to redefine child abuse and neglect in Saudi Arabia according to our culture and religion

The present supportive services to deal with child abuse and neglect in Saudi Arabia are adequate

I prefer resolving a case of child abuse myself rather than reporting it to the police

I am aware of the reporting procedures of child abuse in my hospital in Saudi Arabia

I am willing to report all suspected child abuse cases

I prefer limiting my reporting of child abuse to life-threatening injuries

Child abuse is underreported in Saudi Arabia

The following might be the reasons why child abuse is underreported in Saudi Arabia:

It is not legally mandatory to report child abuse

Reporting might not be good for the sake of the child

Reporting procedures are unclear

Reporting child abuse to authorities is not yet acceptable in our community

Fear of parent response

Do you consider the following acts as abusive toward children?

Burning the child for misbehavior

Locking the child alone at home

Severe beating that leaves marks on the child's body

Parents throwing different objects at the child when angry

Parents who smoke in the presence of the child

Do you consider the following acts as acts of child neglect?

Score

Parents refusing to send the child to school

Parents refusing medical treatment or surgical intervention necessary for their child

Child with severe dental problems, which are not treated

Parents paying no attention to the child's cleanness

Child failing to thrive due to social deprivation

TABLE 4: Child abuse and neglect scoring system

\section{Additional Information}




\section{Disclosures}

Human subjects: Consent was obtained by all participants in this study. Animal subjects: All authors have confirmed that this study did not involve animal subjects or tissue. Conflicts of interest: In compliance with the ICMJE uniform disclosure form, all authors declare the following: Payment/services info: All authors have declared that no financial support was received from any organization for the submitted work. Financial relationships: All authors have declared that they have no financial relationships at present or within the previous three years with any organizations that might have an interest in the submitted work. Other relationships: All authors have declared that there are no other relationships or activities that could appear to have influenced the submitted work.

\section{Acknowledgements}

We would like to extend our thanks to the following individuals: 1. Renad Alghufaily 2. Fatimah Almeathem 3. Abduallh Adakhil 4. Ghadah Alayed 5. Ream Alkhamees

\section{References}

1. Gilbert R, Widom CS, Browne K, Fergusson D, Webb E, Janson S: Burden and consequences of child maltreatment in high-income countries. Lancet. 2009, 373:68-81. 10.1016/S0140-6736(08)61706-7

2. Akmatov MK: Child abuse in 28 developing and transitional countries--results from the Multiple Indicator Cluster Surveys. Int J Epidemiol. 2011, 40:219-227. 10.1093/ije/dyq168

3. Al Eissa M, Almuneef M: Child abuse and neglect in Saudi Arabia: journey of recognition to implementation of national prevention strategies. Child Abuse Negl. 2010, 34:28-33. 10.1016/j.chiabu.2009.08.011

4. Straus MA: Beating the Devil Out of Them: Corporal Punishment in American Families . Transaction Publishers, Piscataway, NJ; 2000.

5. Louwers EC, Affourtit MJ, Moll HA, de Koning HJ, Korfage IJ: Screening for child abuse at emergency departments: a systematic review. Arch Dis Child. 2010, 95:214-218. 10.1136/adc.2008.151654

6. Talsma M, Bengtsson Boström K, Östberg AL: Facing suspected child abuse--what keeps Swedish general practitioners from reporting to child protective services?. Scand J Prim Health Care. 2015, 33:21-26. 10.3109/02813432.2015.1001941

7. Schouten MCM, van Stel HF, Verheij TJM, Houben ML, Russel IMB, Nieuwenhuis EES, van de Putte EM: The value of a checklist for child abuse in out-of-hours primary care: to screen or not to screen. PLoS One. 2017, 12:e0165641. 10.1371/journal.pone.0165641

8. Regnaut O, Jeu-Steenhouwer M, Manaouil C, Gignon M: Risk factors for child abuse: levels of knowledge and difficulties in family medicine. A mixed method study. BMC Res Notes. 2015, 8:620. 10.1186/s13104015-1607-9

9. Demirçin S, Tütüncüler A, Aslan F, Velipaşaoğlu Güney S, Atılgan M, Gülkesen H: The knowledge level and opinions of physicians about the medical and legal procedures related to physical child abuse. Balkan Med J. 2017, 34:140-146. 10.4274/balkanmedj.2015.1195

10. Alsaleem SA, Alsaleem MA, Asiri AM, et al.: Knowledge and attitude regarding child abuse among primary health care physician in Abha, Saudi Arabia, 2018. J Family Med Prim Care. 2019, 8:706-710. 10.4103/ifmpc.jfmpc 44218

11. Dubowitz H, Bennett S: Physical abuse and neglect of children . Lancet. 2007, 369:1891-1899. 10.1016/S0140-6736(07)60856-3

12. Alnasser Y, Albijadi A, Abdullah W, Aldabeeb D, Alomair A, Alsaddiqi S, Alsalloum Y: Child maltreatment between knowledge, attitude and beliefs among Saudi pediatricians, pediatric residency trainees and medical students. Ann Med Surg (Lond). 2017, 16:7-13. 10.1016/j.amsu.2017.02.008

13. Habib HS: Pediatrician knowledge, perception, and experience on child abuse and neglect in Saudi Arabia . Ann Saudi Med. 2012, 32:236-242. 10.5144/0256-4947.2012.236

14. Rad M, Shahravan A, Haghdoost AA: Oral health knowledge, attitude, and practice in 12-year-old schoolchildren in Iran. J Int Soc Prev Community Dent. 2015, 5:419-424. 10.4103/2231-0762.165926

15. Sahebihagh MH, Hosseini SZ, Hosseinzadeh M, Shamshirgaran SM: Knowledge, attitude and practice of community health workers regarding child abuse in Tabriz health centers in 2015-2016. Int J Community Based Nurs Midwifery. 2017, 5:264-274.

16. ALBakr AA, AL-Mari AM, Babidan AA, AlAmin MAM: Knowledge and attitude of primary health care physicians regarding physical child abuse in AL-Khobar city Saudi Arabia 2017. Int J Sci Res. 2018, 7:683692.

17. Kraus C, Jandl-Jager E: Awareness and knowledge of child abuse amongst physicians - a descriptive study by a sample of rural Austria. Wien Klin Wochenschr. 2011, 123:340-349. 10.1007/s00508-011-1579-2

18. Sathiadas MG, Viswalingam A, Vijayaratnam K: Child abuse and neglect in the Jaffna district of Sri Lanka - a study on knowledge attitude practices and behavior of health care professionals. BMC Pediatr. 2018, 18:152. 10.1186/s12887-018-1138-3 